\title{
Hindi nominal suffixes are bimorphemic: A Distributed Morphology analysis
}

\section{Yash Sinha*}

\begin{abstract}
This paper provides a Distributed Morphology (DM) analysis for Hindi nominal (noun and adjectival) inflection. Contra Singh \& Sarma (2010), I argue that nominal suffixes contain two morphemes - a basic morpheme, and a restrictedly distributed additional morpheme. The presence of two different morphemes is especially evident when one compares noun and adjectival inflectional suffixes, which Singh \& Sarma (2010) do not, since they only look at noun inflection. I also show that the so-called adjectival inflectional suffixes are not limited to adjectives, and may occur on nouns, provided the noun is not at the right edge of the noun phrase. On the other hand, the regular noun inflection is only limited to nouns at the right edge of the noun phrase. This is demonstrated using a type of coordinative compound found in Hindi. Then, I take the fact that nouns can take either the regular noun inflection or the so-called "adjectival" inflection as motivation for a unified analysis for both sets of suffixes. I demonstrate that after undoing certain phonological rules, the difference between the "adjectival" and regular noun inflectional suffixes can be summarized by saying that the additional morpheme only surfaces in the regular noun inflectional suffixes. Finally, I provide vocabulary entries and morphological operations that can capture the facts about the distribution of the various basic and additional morphemes.
\end{abstract}

Keywords. Hindi; noun inflection; adjectival inflection; Distributed Morphology

1. Introduction. Hindi nominals (nouns and adjectives) inflect for case, number and gender. In this paper, I argue that these nominal inflectional suffixes contain up to two morphemes. I discuss the forms and distributions of these morphemes, and then present a Distributed Morphology (Halle \& Marantz 1993, 1994 - henceforth DM) analysis that can account for them.

While several works discuss Hindi nominal inflectional morphology (including Shapiro 1989, Kachru 2006), to my knowledge, there exists only one other account of Hindi nominal morphology within the DM framework (Singh \& Sarma 2010, henceforth S\&S).

S\&S only look at noun inflection, and do not consider adjectival inflection. The current analysis differs from S\&S's in claiming that Hindi noun inflectional suffixes can contain up to two morphemes. This difference arises partially (but not entirely) because there are certain segments that $S \& S$ treat as part of the noun, that I argue should be treated as part of the inflectional suffix. More specifically, they treat the $-i$ ending on a subset of feminine nouns as part of the noun. However, the $-i$ is better analyzed as an inflectional morpheme. This will be especially evident when we look at the adjectival inflection paradigm, which S\&S do not consider.

This paper is organized as follows. In Section 2, I present the noun and adjectival inflection paradigms, and discuss their distribution. I show that the so-called adjectival inflection suffixes can sometimes appear on nouns as well, and use that to conclude that adjectival and noun

\footnotetext{
*I would like to thank Karlos Arregi for his mentorship throughout my work on this project. I would also like to thank the anonymous reviewers, and the attendees at LSA 2018 Annual Meeting (especially Rajesh Bhatt), for their comments. All errors are my own. Author: Yash Sinha, University of Chicago (yashsinha@uchicago.edu)
} 
inflection should receive a unified treatment. In Section 3, I show that some of the noun inflectional suffixes can be decomposed into the corresponding adjectival inflectional suffix, followed by an additional morpheme. This supports the main claim of the paper: that Hindi nominal suffixes are bimorphemic. Section 4 gives the vocabulary entries and morphological operations needed to account for the distribution of the various morphemes in the nominal inflectional paradigms. Section 5 concludes this paper.

2. Two paradigms of nominal inflection. Let us start the discussion with noun inflection. Hindi nouns inflect for number (singular or plural) and case (direct or oblique). Direct case corresponds to what has been called the nominative or absolutive case in the literature on Hindi. Oblique case refers to the form of the noun that occurs with enclitics (such as the ergative $=n e$ or the DOM $=k o$ ). Nouns belong to one of two genders - masculine or feminine.

Under the current analysis, all nouns and adjectives belong to two inflectional classes, which I will call Class I and Class II. For nouns, these classes correspond to the two classes that Shapiro (1989) also posits for Hindi nouns. He does not use the Class I/Class II terminology for adjectives, however. He classifies adjectives as declinable and indeclinable. I claim that declinable adjectives are Class I adjectives, and indeclinable adjectives are Class II adjectives. Note that I am saying that the categories Class I and II for adjectives are the same as the categories Class I and II for nouns. For both adjective and nouns, these inflectional classes are represented as features on roots.

Before moving on, consider how the inflectional classes I am using correspond to S\&S's inflectional classes for nouns. Table 1 shows this.

\begin{tabular}{ccc} 
& Feminine & Masculine \\
\hline Class I & Class B & Class D \\
Class II & Class C & Class E \\
\hline
\end{tabular}

Table 1: Correspondence between S\&S's inflectional classes and the ones used here

S\&S conflate gender and inflectional class, which is why each of the inflectional classes I posit corresponds to two inflectional classes $S \& S$ posit - one for masculine, and one for feminine. They have Classes B \& D for the Class I here, and Classes C \& E for Class II.

I do not consider S\&S's Class A as an inflectional class. Their Class A is characterized by the fact that it does not take any suffixes at all. As they themselves note, Class A is comprised of uncountable nouns. In fact, none of the nouns they mention (including ag ('fire), krodh ('anger'), pyas (thirst)) can appear in the plural. Consider, for example, krodh ('anger'). Just like in English, it can only occur in the singular. Consider (1) and (2) below.

$\begin{array}{lllll}\text { mer-a } & \text { krodh } & \text { mojhe } & \text { pagəl kər-t-a } & \text { th-a } \\ \text { 1.POSS-M.SG } & \text { anger } & \text { 1.DAT } & \text { crazy make-IMPF-M.SG } & \text { PST-M.SG } \\ \text { "My anger used to make me crazy." } & & \\ \text { *mer-e } & \text { krodh } & \text { mojhe } & \text { pagəl kər-t-e } & \text { th-e } \\ \text { 1.POSS-M.PL } & \text { anger } & \text { 1.DAT } & \text { crazy make-IMPF-M.PL } & \text { PST-M.PL }\end{array}$
Intended: "My angers used to make me crazy."

The inflections on the possessive determiner and the verb show that these nouns cannot be pluralized. These nouns also do not take any additional suffix in the singular, but that is also true for all Class II nouns (i.e. S\&S's Class C and E). Thus, the only thing that sets Class A nouns 
apart from Class $\mathrm{C} \& \mathrm{E}$ nouns, is that they cannot be pluralized, and this follows from their semantics. We do not need to posit a separate inflectional class for them.

Now, consider the noun inflection paradigm in Table 2, which shows the different suffixes different classes of nouns take depending on their gender, number and case.

\begin{tabular}{lllll} 
& Class I & \multicolumn{3}{c}{ Class II } \\
\hline & FEM. & MASC. & FEM. & MASC. \\
SG. DIR. & $-i$ & $-a$ & $-\varnothing$ & $-\varnothing$ \\
SG. OBL. & $-i$ & $-e$ & $-\varnothing$ & $-\varnothing$ \\
PL. DIR. & $-I y \tilde{a}$ & $-e$ & $-\tilde{e}$ & $-\varnothing$ \\
PL. OBL. & $-r y \tilde{o}$ & $-\tilde{o}$ & $-\tilde{o}$ & $-\tilde{o}$ \\
\hline
\end{tabular}

Table 2: Noun inflectional suffixes

In Class I feminine nouns, S\&S do not include the $-i$ (and its phonologically conditioned allomorph $-I$ at the beginning of the plural suffixes) as part of the inflectional suffixes. A reasoning for this could be that since all forms of these nouns end in $-i$, the $-i$ is actually part of the noun, rather than the inflectional suffix. However, if we consider the suffixes adjectives take, we see that the $-i$ also occurs with Class I adjectives. The adjectival inflectional suffixes are given in Table 3. In Hindi, adjectives precede the nouns they modify, such that the noun is the right most element of a noun phrase.

\begin{tabular}{lllll} 
& Class I & \multicolumn{3}{c}{ Class II } \\
\hline SG. DIR. & FEM. & $-i$ & MASC. & FEM. \\
SG. OBL. & $-i$ & $-a$ & $-\varnothing$ & $-\varnothing$ \\
PL. DIR. & $-i$ & $-e$ & $-\varnothing$ & $-\varnothing$ \\
PL. OBL. & $-i$ & $-e$ & $-\varnothing$ & $-\varnothing$ \\
\hline
\end{tabular}

Table 3: Adjectival inflectional suffixes

Unlike nouns, adjectives do not have inherent gender. They inflect for gender based on the noun they modify, and for Class I adjectives, the feminine suffix $-i$ is in complementary distribution with the masculine suffixes $-a /-e$. For example, if the adjective "yellow" modifies a feminine noun, its form is pil-i, but if it modifies a masculine noun, its form is pil-a/e. Thus, the feminine marking suffix $-i$ cannot be part of the adjectival root, unless we want to posit two separate adjectives, one for the masculine and one for the feminine. Therefore, it is best to analyze $-i$ as part of the inflectional suffix, at least for adjectives.

One might insist that adjectival and noun inflection suffixes are different from each other. However, this view in not tenable in light of the fact that sometimes nouns can also take the socalled adjectival inflectional suffixes.

Adjectival suffixes may occur on nouns as well, as long as the noun is inside a noun phrase, and not at its right edge. Consider (3), which features a coordinative compound that contains two nouns next to each other (X-Y, with the meaning "X and Y"). In such compounds, the first noun takes the adjectival inflectional suffix rather than the noun inflection suffix. 

(3) kott-e - bill-ıуõ ko
dog-M.PL.OBL. - cat-F.PL.OBL DAT
"to dogs and cats"
(*kutt-õ - bill-ıyõ ko)

Here, the DP is in oblique case. Both nouns are plural, but the first one is masculine, and the second one is feminine. Masculine plural oblique nouns are expected to take - $\tilde{o}$, but here the first noun takes $-e$, which is what Class I adjectives take for masculine plural oblique. Note that this cannot be accounted for by saying that the first noun necessarily occurs in the direct case, since the masculine plural direct suffix for nouns is also $-e$. Consider (4), where both nouns are in the singular.

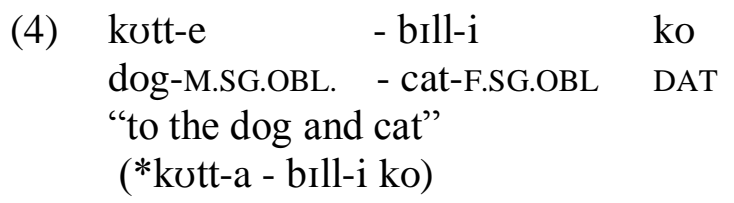

If the first noun had to necessarily occur in the direct case, then it would take the suffix - $a$. This is not the case. Therefore, I conclude that the first noun in a coordinative compound must take the so-called adjectival inflectional suffix.

Given the fact that nouns can take both "adjectival" suffixes from Table 3 or the regular noun suffixes from Table 2, it is desirable to have a unified analysis for both sets of suffixes. Of course, we also need to distinguish between the two since the two have different forms and different distributions. We will consider how the forms are related to each other in the next section, but as far as their distributions go, we can say the following: the "adjectival" suffixes (i.e. from Table 3 ) are found inside a noun phrase (that is, on adjectives and on the first noun in a compound), while the regular noun suffixes (i.e. from Table 2) are found at the right edge of the noun phrase.

3. Decomposing the noun inflectional suffixes. Let us turn our attention to the relation between the forms of the suffixes in Tables 2 and 3, reproduced as Tables 4 and 5 respectively.

\begin{tabular}{lllll} 
& Class I & \multicolumn{5}{c}{ Class II } \\
\hline & FEM. & MASC. & FEM. & MASC. \\
SG. DIR. & $-i$ & $-a$ & $-\varnothing$ & $-\varnothing$ \\
SG. OBL. & $-i$ & $-e$ & $-\varnothing$ & $-\varnothing$ \\
PL. DIR. & $-I y \tilde{a}$ & $-e$ & $-\tilde{e}$ & $-\varnothing$ \\
PL. OBL. & $-r y \tilde{o}$ & $-\tilde{o}$ & $-\tilde{o}$ & $-\tilde{o}$ \\
\hline
\end{tabular}

Table 4: Noun inflectional suffixes

\begin{tabular}{lllll} 
& Class I & \multicolumn{3}{c}{ Class II } \\
\hline SG. DIR. & FEM. & $-i$ & MASC. & FEM. \\
SG. OBL. & $-i$ & $-a$ & $-\varnothing$ & $-\varnothing$ \\
PL. DIR. & $-i$ & $-e$ & $-\varnothing$ & $-\varnothing$ \\
PL. OBL. & $-i$ & $-e$ & $-\varnothing$ & $-\varnothing$ \\
\end{tabular}

Table 5: Adjectival inflectional suffixes 
The suffixes in Table 4 can be decomposed into the suffixes in Table 5, followed by an additional morpheme in some situations. To see that, we need two phonological rules to account for some changes to the phonological shape of the suffixes. These are in (5) and (6).

$$
i \rightarrow \mathrm{ryl} \text { [V }_{\text {-front }]}
$$

(5) has been posited in prior literature on Hindi (in a slightly different formulation, in Elizarenkova 1988), and can be independently motivated in the language. For example, an application of (10) can be seen in the word prya $\leftarrow p i-a=p i$ (drink) $+-a$ (M.SG.PERF). Since both

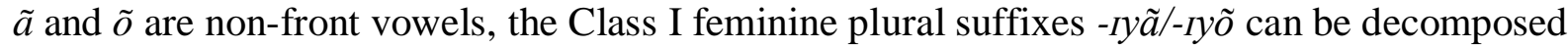
into $-i+-\tilde{a} / \tilde{o}$.

The other rule is given in (6).

$e \rightarrow \emptyset /\left[\mathrm{V}_{+\mathrm{back}}\right]$

I am not aware of any previous work that mentions this rule, but this rule can account for some minor irregularities in the Hindi verbal inflection system, which is otherwise fairly regular. Consider the verb roots $d e$ ('give') and le ('take'). With the 2.PL suffix, - $o$, they give $d o$ and $l o$ instead of the expected *deo and *leo. Furthermore, to my knowledge, there are no surface forms that have the sequence $e o$. (6) can account for why the $-e$ does not surface in the plural oblique for Class I masculine nouns in the regular noun paradigm, even though it does in the "adjectival" paradigm. The $-\tilde{o}$ is only present in the regular noun paradigm, and thus (6) only applies in the regular noun paradigm, and not the adjectival paradigm.

Table 6 shows the regular noun inflectional suffixes from Tables 2 and 4, but this time decomposed into their constituent morphemes.

\begin{tabular}{lllll}
\multicolumn{1}{c}{ Class I } & \multicolumn{3}{c}{ Class II } \\
\hline SG. DIR. & FEM. & $-i$ & MASC. & FEM. \\
SG. OBL. & $-i$ & $-e$ & $-\varnothing$ & $-\varnothing$ \\
PL. DIR. & $-i+-\tilde{a}$ & $-e$ & $-\varnothing$ & $-\varnothing$ \\
PL. OBL. & $-i+-\tilde{o}$ & $-e+-\tilde{o}$ & $-\varnothing+-\tilde{e}$ & $-\varnothing$ \\
\hline
\end{tabular}

Table 6: Noun inflectional suffixes decomposed

Comparing Tables 5 and 6, it becomes clear that the regular noun inflection paradigm just consists of additional morphemes stacked on top of the morphemes that constitute the "adjectival" suffixes. The morphemes that constitute the "adjectival" suffixes, I will call "basic morphemes." The morphemes that are stacked on top of basic morphemes in the regular noun suffixes, I will call "additional morphemes". Thus, Hindi nominal suffixes contain basic morphemes, and may contain another additional morpheme following that. That is, they are bimorphemic.

Let us now reframe the generalization about the distribution of the two paradigms in terms of basic and additional morphemes. Basic morphemes occur on all nouns and adjectives, regardless of where they occur in a noun phrase. Additional morphemes only occur at the right edge of the noun phrase, and follow the basic morpheme on the last noun in a noun phrase. 
4. Analysis within DM. I begin by positing the internal syntactic structure for the DPs discussed above. A DP that only contains a noun has the structure in (7). The root is headed by a nominalizing head, $n$, which in turn is headed by a $\mathrm{D}$ head.

(7)

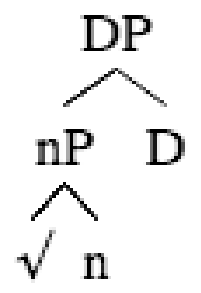

When we have an adjective modifying the noun, the structure we have is as shown in (8). The adjectival root is followed by an adjectivalizing head $a$ to form the $a \mathrm{P}$, which attaches as the specifier of $n$. Note that in this structure, it is the noun, and not the adjective that is adjacent to the $\mathrm{D}$ head. This will be crucial when explaining the syntactic distribution of additional morphemes.

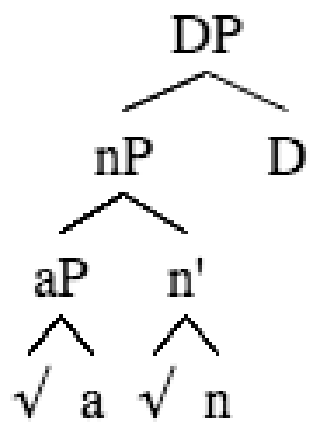

Finally, when we have coordinative compounds, the structure is as shown in (9). I do not commit to a particular theory of coordination. For ease of exposition, I have chosen the structure below, but this analysis holds for any coordinative compound structure we assume, as long as the second noun in the compound is adjacent to the D head and first noun is not. Again, this will be crucial in explaining the distribution of additional morphemes. 


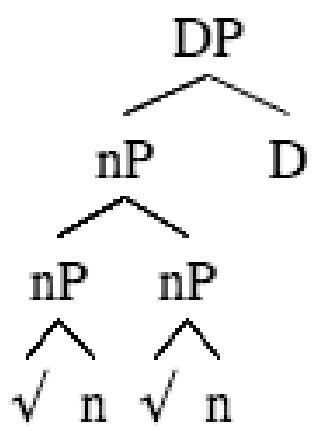

The distribution of gender, number and case features are as follows: gender and number features originate on the $n$ head, while case features start on the D head ${ }^{1}$. However, Agree is responsible for transmitting some of these features to other heads. In particular, the adjectivalizing head $a$ receives gender and number features from the head noun's $n$, and case features from D. Furthermore, $n$ also receives case features from $\mathrm{D}$ via Agree. Therefore, by the time of vocabulary entry, both $a$ and $n$ have gender, number and case features, and D has case features. We also have [Class I] and [Class II] features, located on all roots.

Basic morphemes are spelt out on $a$ and $n$ heads. The relevant vocabulary entries for these are given in (10)-(13).

(10) $\quad[] /[$ Class II $] \rightarrow \varnothing$

(11) $[$ feminine $] /[$ Class $\mathrm{I}] \rightarrow-i$

(12) [masculine, singular]/[Class I] $\rightarrow-a$

(13) []$/[$ Class I] $\rightarrow-e$

These entries can partially explain the distribution of basic morphemes. Class II nominals always take the null morpheme regardless of the features involved, as predicted by (10). For Class I feminine nominals, we always get $-i$ as the basic morpheme, which is what (11) would predict. Class I masculine nominals are a little trickier to explain. We would expect them to take $-a$ in the singular, leaving the underspecified $-e$ for the plural (since no entry from (11) or (12) would be applicable in the masculine plurals). However, this is only partially what we see. Unexpectedly, in the singular oblique, Class I masculines take the underspecified $-e$ instead of the expected $-a$.

One possible remedy to this would be to specify (12) for direct case as well i.e. restate (12) to read something like: [masculine, singular, direct]/[Class I] $\rightarrow-a$. This would rule out $-a$ in the oblique case, leaving the underspecified $-e$ for it. The reason I do not take this approach is because of verb agreement. When verbs show gender/number agreement, they use the same three basic morphemes as Class I nominals ( $-a$ for masculine singular, $-e$ for masculine plural and $-i$ for feminines ${ }^{2}$ ). Assuming that these verb agreement morphemes are the same as the nominal suffix morphemes we have been dealing with, and verbal agreement does not involve case features, we cannot have the vocabulary entries (11)-(13) be specified for case features.

\footnotetext{
${ }^{1}$ Or more accurately, they have been assigned to the D head from a higher enclitic or a head in the verbal domain.

${ }^{2}$ And an additional morpheme (nasalization) following the $-i$ in the feminine plural, in certain syntactic contexts. See Bhatt \& Keine (2017)
} 
Therefore, I do not take this approach. The approach I will be taking is using impoverishment rule (14).

(14) [singular, oblique] $\rightarrow$ [oblique]

That is, the feature [singular] is deleted in oblique contexts. Therefore, since [singular] is deleted in the oblique case, the morpheme $-a$ becomes ineligible for insertion in Class I masculine singular obliques, leading to the insertion of the underspecified $-e$. Thus, we can account for the distribution of all basic morphemes.

Turning to additional morphemes, these are limited to plural contexts. Furthermore, they are only found in either feminine or oblique contexts i.e. they are not found in the masculine direct. And, as we noted earlier, there is also a syntactic restriction on their distribution: they only occur at the right edge of a noun phrase.

I posit two fission rules, (15) and (16) that put [plural] on a separate node, when this feature co-occurs with [feminine] or [oblique].

(15) $[$ feminine, ..., plural $] \rightarrow$ [feminine, ...] [plural]

(16) [oblique, ..., plural] $\rightarrow$ [oblique, ...] [plural]

The additional morphemes are realizations of this newly created [plural] node, which is why they are only limited to plurals. These fission rules are also able to account for why these morphemes are only found in the feminine plural or oblique plural, and not in the masculine direct plural. In the masculine direct plural, there is no fission, and the node for the additional morpheme is not created. The vocabulary entries for additional morphemes are given in (17)(19).

(17) [plural]/_D

(18) [plural]/_D[direct], [Class I] $\rightarrow-\tilde{a}$

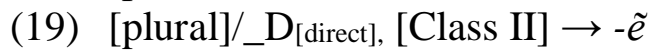

Recall that $\mathrm{D}$ also has case features on it. This is what is meant by the subscript indicating the case features next to D. These entries explain the distribution of additional morphemes. In the oblique case, the [plural] is realized as $-\tilde{o}$, according to (17). In the direct case, we get different

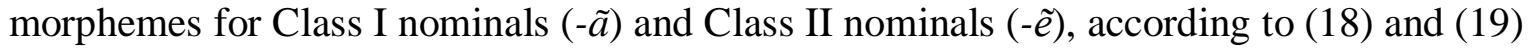
respectively.

The reason that the additional morpheme is only found at the right edge of a noun phrase is because entries (17)-(19) are restricted to contexts where the [plural] node is immediately followed by the D head. This only occurs for the final noun in a noun phrase. The $a$ head on an adjective and the $n$ head on the first noun of a coordinative compound are not adjacent to the D head. Hence, these elements do not have an additional morpheme attached to them.

Note that the fission that creates an additional plural node occurs everywhere where the relevant features are present. It is only the insertion of vocabulary items in (17)-(19) that is limited to the right edge of the noun phrase. Thus, we are also able to account for the distribution of additional morphemes in the Hindi nominal suffix paradigms.

5. Conclusion. In this paper, I have shown that Hindi nominal suffixes can contain up to two morphemes (a basic morpheme, and an additional morpheme), though the bimorphemic nature of some of these suffixes might be obscured by certain phonological rules. Moreover, the additional morpheme surfaces only in certain contexts. Morphologically, it only surfaces in the direct plural 
for feminine nouns, and in the oblique plural for all nouns. This was derived with two fission rules that create an additional plural node (which is where the additional morpheme is realized) in feminine and oblique contexts. Syntactically, the additional morpheme is limited to the right edge of the noun phrase. This restriction was accounted for by positing that the additional morpheme is only realized when the plural node is immediately adjacent to the D head. I also posited vocabulary entries and morphological operations, that were able to account for the distribution of basic and additional morphemes.

Part of the reason we were able to see that Hindi noun suffixes are bimorphemic is because there were some morphemes that $\mathrm{S} \& S$ considered as part of the noun, which we considered as part of the inflectional suffix. This, in turn, was a result of comparing the noun suffixes to the adjectival suffixes. Furthermore, by looking at coordinative compounds, we were able to see that the distribution of "adjectival" and the regular noun suffixes are related to each other; their distribution does not have to do with the category of noun or adjective, but is rather concerned with the syntactic locus at which the morpheme is realized.

From this paper, I can foresee two lines of future work. Within the language, it would be interesting to see if this analysis can be extended to verbal agreement gender/number morphemes, that as I mentioned earlier, are quite similar in form. Bhatt \& Keine (2017) provide a DM analysis for these morphemes, but the technicalities of their proposal differ in some ways from mine. I leave for future research the work of seeing if the two analyses could be made compatible.

Cross-linguistically, this paper also raises the question of what an inflectional class is, and how it should be represented. In this paper, we saw that the allomorphy that the category of inflectional class is supposed to capture can be accounted for by positing a feature on the root, that triggers allomorphy on the adjacent morpheme. Can we account for all the effects of inflectional classes in other languages in this way? Relatedly, a lot of languages are like Hindi, in the sense that certain inflectional classes have characteristic endings based on gender, but certain others do not. Can those data be accounted for in the same way as has been done for Hindi here, by assuming that these characteristic endings are inflectional morphemes? I leave all these questions for future work.

\section{References}

Bhatt, Rajesh \& Stefan Keine 2017. Tense and the realization of the feminine plural in HindiUrdu. In G. Sengupta, S. Sircar, M. Gayathri \& R. Balusu (eds.), Perspectives on the Architecture and Acquisition of Syntax: Essays in Honour of R. Amritavalli. Dodrecht: Springer. http://www-bcf.usc.edu/ keine/papers/BhattKeine_tense.pdf

Elizarenkova, T. Y. 1988. Morphophonology of Hindi. In M. S. Andronov and B. P. Mallik (eds.), Linguistics: A Soviet Approach. 367-386. Calcutta: Indian Journal of Linguistics.

Halle, Morris and Alec Marantz 1993. Distributed Morphology and the pieces of inflection. In K. Hale and S. J. Keyser (eds.), The View from Building 20. 111-176. Cambridge, MA: MIT Press. http://home.uni-leipzig.de/muellerg/dm8.pdf

Halle, Morris and Alec Marantz 1994. Some Key Features of Distributed Morphology. In A. Carnie, et al. (eds.), Papers in Phonology and Morphology. 275-288. MITWPL 21. http://ling.auf.net/lingbuzz/000086 
Kachru, Yamuna 2006. Hindi. Amsterdam: John Benjamins.

Shapiro, Michael C. 1989. A primer of modern standard Hindi. Delhi: Motilal Banarsidass.

Singh, Smrithi \& Vaijayanthi M. Sarma 2010. Hindi Noun Inflection and Distributed Morphology. International Conference on Head-Driven Phrase Structure Grammar 17. 307-321. http://web.stanford.edu/group/cslipublications/cslipublications/ HPSG/2010/singh-sarma.pdf 